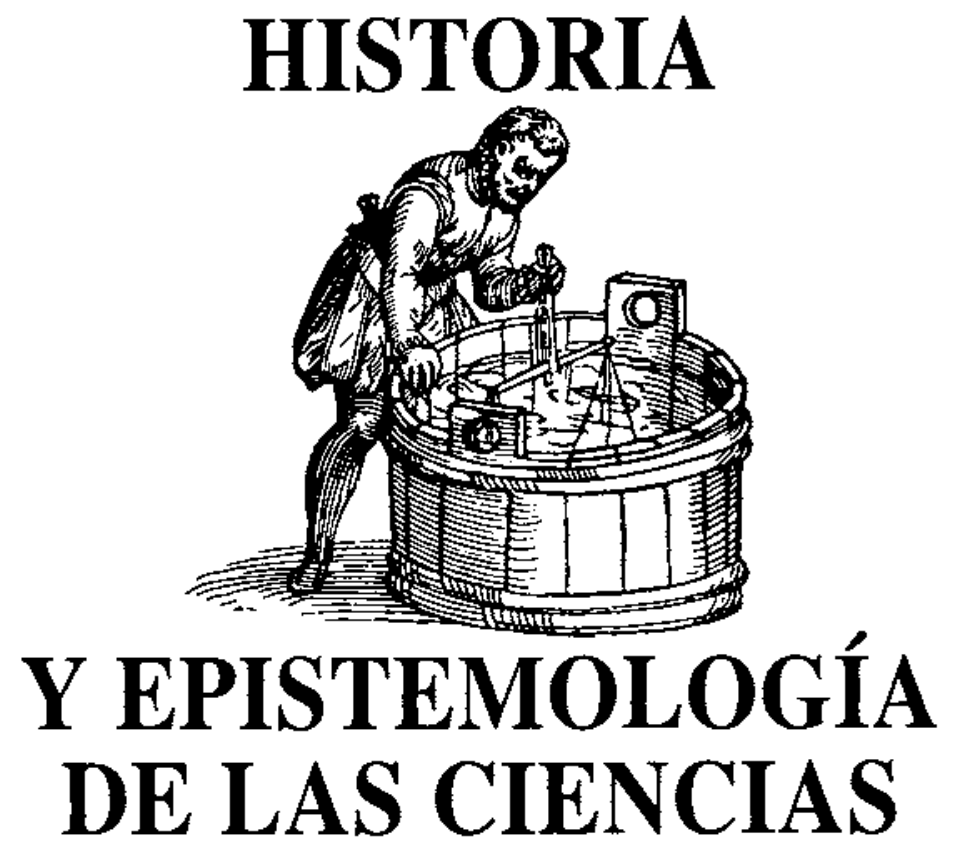

\title{
LA PERTINENCIA DE LA HISTORIA EN LA ENSEÑANZA DE CIENCIAS: ARGUMENTOS Y CONTRAARGUMENTOS
}

LOMBARDI, O.I.

Gabinete de Desarrollo de Metodologías de la Enseñanza. Facultad de Ingeniería.

Universidad de Buenos Aires. Av. Paseo de Colón, 850, $2^{\circ} .1063$ Buenos Aires. Argentina.

\section{SUMMARY}

Adding up to the debate about the possibility of a historical view in science teaching, the present article frames some responses to the criticisms that, on the basis of the need of simplification and reconstruction of history, object its intrusion in science education. In that sense, the essentially constructive character of history and, particularly, of history of science is appointed, where the epistemological position of the historian acquires special relevance. The multiplicity of historical stories is a constituent characteristic of history and, thus, it does not represent an obstacle in sciences teaching.

\section{CRÍTICAS AL ENFOQUE HISTÓRICO}

En la actualidad, existe un consenso casi unánime entre los investigadores en educación acerca de la relevancia de la perspectiva histórica en la formación científica. En los úftimos años se ha verificado una progresiva incor- 
poración de la historia de la ciencia tanto a la teoría como a la práctica de la enseñanza de ciencias. De este modo, se produce un acercamiento entre áreas del conocimiento tradicionalmente consideradas ajenas entre sí según una antigua clasificación que separa ciencias de humanidades.

Sin embargo, en un clima que propicia la comunicación entre ciencia natural, historia y filosofía, algunas voces se alzan para presentar ftiertes críticas al programa de introducir la historia de las ciencias en la enseñanza de ciencias. Una de estas voces es la de Martin Klein, quien sostiere que todo intento de presentar los contenidos científicos desde una perspectiva histórica implica «seleccionar, organizar y presentar estos materiales históricos no históricamente, quizás incluso antihistóricamente» (Klein, 1972). De acuerdo con el autor, existe una diferencia esencial entre la perspectiva del físico y la dei historiador: Así, «es difícil imaginar la combinación entre la rica complejidad de hechos a los que el historiador aspira, con la versión simple y precisa que el físico busca" (Klein, 1972). Sobre la base de estas consideraciones, Klein concluye que toda introducción de la historia en la enseñanza de ciencias sólo tiene la posibilidad de hacer uso de una historia simplificada y recortada, esto es, una mala historia; ante tal alternativa, prefiere prescindir totalmente de la historia.

Sobre la misma línea argumentativa, otra objeción levantada contra la relevancia de la historia en la enseñanza de ciencias es la presentada por Whitaker, quien señala que la historia utilizada en los cursos de ciencia es, en realidad, una casi historia pues constituye «el resultado de numerosos libros de autores que han sentido la necesidad de dar vida a sus explicaciones [...] con un poco de contenido histórico, pero que de hecho han reescrito la historia acomodándola paso a paso con la física» (Whitaker, 1979). Evaluando diversos trabajos, Whitaker concluye que muchos relatos históricos son construidos deliberadamente, o bien con fines pedagógi $\cos$, o bien con el objetivo de apoyar la concepción epistemológica del autor.

Ambas críticas, si bien similares en su espíritu, apuntan a dos aspectos diferentes del problema del conocimiento histórico: Ja cuestión de la simplificación de la historia y la cuestión de la interpretación de los hechos históricos. A continuación se abordarán con mayor detalle ambos aspectos.

\section{EL PROBLEMA DE LA SIMPLIFICACIÓN}

El argumento de Klein parece apuntar a la tergiversación que implicaría una historia simplificada, al no tomar en consideración la rica complejidad de los hechos del pasado. Según esta perspectiva, toda omisión constituye una «fałla» o «defecto» del relato histórico. La «mala historia", simplificada y recortada, se opone así a la «buena historia», que parece requerir, por lo tanto, un carácter completo y exhaustivo.
Frente a esta objeción surge de inmediato la pregunta: ¿Es posible aspirar a la «completitud» y la exhaustividad en la producción histórica? Si bien las nuevas informaciones acerca del pasado - bajo la forma de testimonios, documentos, etc.- contribuyen a enriquecer el bagaje empírico mediante el cual el historiador construye su propio relato, elto no implica la irrelevancia del trabajo de quienes aún no contaban con tales datos. No es posible suponer que la legitimidad de la investigación histórica se funde en el carácter completo de los datos sobre los que se basa, pues tal "completitud» teórica es absolutamente inalcanzable: el pasado constituye una fuente inagotable de información, y la tarea del historiador consiste, precisamente, en organizar y seleccionar tal información con el objetivo de hallar un hilo conductor a través del cual los meros hechos cobren su sentido.

La visión de una historia cuya misión no consiste en la mera recapitulación exhaustiva de datos «imparciales» sino en la elaboración de un relato coherente y significativo que permita explicar acontecimientos del pasado, no es en modo alguno reciente. Tal tesis recibe su fundamento filosófico con Hegel y su concepción del proceso de la historia como la manifestación del despliegue de la razón en etapas o momentos sucesivos (Hegel, $1975,1985)$. Este aspecto de la doctrina de Hegel fue ampliamente recogido por numerosos autores, quienes, más allá de su mayor o menor adhesión al conjunto đel sistema hegeliano, adoptaron la idea de la racionalidad de la historia. Un representante destacado de esta corriente de pensamiento fue el filósofo francés del siglo $X I X$ Victor Cousin (1846), quien, bajo influencia hegeliana, adoptó una filosofía de Ia historia basada en la legalidad subyacente a los acontecimientos y personajes históricos: la historia no es una mera sucesión de hechos sino la expresión de las leyes intrínsecas que rigen el devenir, tanto universal como de cada pueblo. A la luz de esta doctrina, Ia tarea del historiador ya no consiste en acumular información del modo más completo y ex haustivo posible, sino en descubrir tales leyes subyacentes. La amplia influencia de Cousin alcanzó incluso el círcuto intelectual latinoamericano. Ya en 1845 , Domingo $F$. Sarmiento expresaba en su colorido estilo la necesidad de hallar el hilo conductor de la historia: «Los hechos están ahí consignados, clasificados, probados, documentados; fáltales, empero, el hilo que ha de ligarlos en un solo hecho, el soplo de vida que ha de hacerlos enderezarse a todos a un tiempo a la vista del espectador, y convertirlos en cuadro vivo" (Sarmiento, 1993, p. 53).

Si estas consideraciones son válidas en el ámbito de la investigación histórica, lo son aún más en el caso de la enseñanza de historia. Todo relato historico orientado a la enseñanza debe necesariamente seleccionar algunos hechos considerados relevantes a la Iuz del sentido que se desea brindar a tal relato. Si se pretendiera que los alumnos abordasen la historia con la profundidad y el detalle del historiador, seguramente sería imposible avanzar más allá del primer contenido presentado en clase. En otras palabras, si la exhaustividad en la consideración de los hechos del pasado fuese realmente un requisito indispensable para obtener una «buena historia», estaríamos siempre enseñando «mala historia». 
Dado que el ideal de una historia exhaustiva y no simplificada constituye un mito inalcanzable, surge entonces una nueva pregunta: ¿Sobre la base de qué criterio se efectúa el recorte de los hechos del pasado?

La discriminación entre los hechos considerados relevantes y aquéllos que no revisten una importancia central en el devenjr historico se encuentra fuertemente determinada por la interpretación adoptada por el autor. Esto nos conduce de inmediato a la segunda cuestión: el problema de la reconstrucción de la historia.

\section{EL PROBLEMA DE LA RECONSTRUCCIÓN}

En un célebre texto publicado a principios de la década de 1950, Herbert Butterfield (1951) inauguró un debate que aún sigue vigente: apropiándose de un término caracteristico de la historia constitucional inglesa, denominó historiografía whig al enfoque de la historia de la ciencia que suelen adoptar muchos textos y profesores y que, en síntesis, consiste en imponer al pasado los patrones del presente y evaluar la ciencia de épocas preteritas a la luz y con referencia al conocimiento actual.

Quienes, en el siglo XIX, redactaron la historia de Inglaterra desde la perpectiva whig, concibieron la «conquista de la libertad» como un proceso acumulativo que se remonta a la Carta Magna de 1215 y adquiere su mayor significación en el siglo XVII, en el cual los adherentes al partido whig son considerados amantes de la Iibertad y sus adversarios, los tories, enemigos de ella. La mayoría de las «historias patrias», dentro y fuera de Inglaterra, adoptan este esquema simplista y lo consagran como mito.

Trasladada a la historia de la ciencia, la perspectiva whig implica considerar, en cada momento histórico, únicamerte los elementos relevantes para el pensamiento científico subsiguiente, lo cual permite clasificar autores, obras e instituciones en dos categorías excluyentes: "progresistas» y «conservadores». La aplicacion de tal criterio eliminará de la historia de la ciencia las teorías que resulten «erróneas» a la luz de la ciencia posterior, salvo que se las analice para señalar el carácter retrógrado de quienes las sostuvieron; desechará, además, los factores que hoy consideramos no científicos o irracionates. Dado que incluso los grandes científicos consagrados por la historiografía whig cayeron en tales «errores», éstos se ignoran atribuyéndolos a eventuales deslices del genio; ejemplo de ello es el silencio acerca de los fuertes intereses alquímicos de Newton (Dobbs, 1975, 1991; Figala, 1977). Debido a su carácter lincal, la historiografía whig se encuentra estrechamente relacionada con la tesis de la continuidad acumulativa del conocimiento científico, sostenida, entre otros, por los historiadores de la ciencia Pierre Duhem (1959) y George Sarton (1970).

La principal crítica que se dirige a la historiografía whig se centra en su linealidad y su anacronismo: si bien el relato histórico así construido puede resultar atractivo en la medida en que pone el ếnfasis en la progresión racional de ideas, proporciona una visión distorsionada de la historia. Según esta crítica, la historiografía anacrónica conduce a la tentación de escribir la historia «hacia atrás», dando como resultado una historia «teleológica» de las ciencias (Kuhn, 1970), en la cual «cada etapa de la ciencia conduce a la etapa siguiente - a la que prepara-, y prolonga la etapa anterior $-a$ la que consumaw (Fichant y Pécheux, 1971, p. 91). Los autores que rechazan la perspectiva whig proponen, en su lugar, un ideal diacrónico que aborde los acontecimientos del pasado en términos del contexto-creencias, teorías, métodos, etc.- vigente en la época considerada.

Esta antinomia historiográfica conduce a una muy diferente valoración de los episodios del pasado. El historiador whig substancializa la ciencia como entidad autónoma y suprahistorica; en relación con ella pueden evaluarse logros y fracasos del pasado. Pero tal valoración carece de sentido para la historiografía antiwhig, que prohibe interpretar el pasado a la luz del conocimiento presente y permite evalıar logros y fracasos sólo en relación con el contexto de la época en que tales acontecimientos sucedieron. En ambos casos, Ios relatos históricos resultantes suelen ser radicalmente diferentes.

La influencia de la crítica antiwhig ha sido decisiva para volcar a muchos historiadores hacia la tesis del contextualismo. Bajo la guía del ideal diacrónico, se han producido numerosos trabajos dotados de una gran riqueza y una razonable dosis de objetividad. Sin embargo, un enfoque diacrónico estricto es utopico; todos nos encontramos inmersos en nuestro propio contex to; nadie escapa a su tiempo ni puede prescindir de los «anteojos del presente» mediante los cuales accede a la mirada histórica. Algunos autores se preguntan, incluso, hasta qué punto el diacronismo estricto, de ser posible, sería deseable, pues implica el riesgo de "anticuarismo» y de que el conocimiento histórico se torne inaccesible salvo para unos pocos especialistas (Boido, 1992).

El problema de los distintos enfoques historiográficos en historia de la ciencia ha sido abordado en profundidad por Gastón Bachelard y por otros pensadores inspirados por sus trabajos, como Michel Fichant y Michel Pécheux. Estos autores critican duramente la historia teleológica: «La teleología -porque instaura un vínculo retrospectivo, exterior a los términos que une- no hace más que recubrir el azar y la contingencia radical de la sucesión de los hechos, de Ios resultados acumulados por la ciencia» (Fichant y Pécheux, 1971, p. 91); sin embargo, el mero interés «anticuarista» por el pasado de la ciencia no constituye en absoluto una verdadera historia de la ciencia. Según Bachelard, es necesario adjuntar a la historia de los hechos una historia evaluadora, cuyos criterios de valor se fundan en la ciencia actual (Bachelard, 1951b); esta «historia recurrente» implica una «asimilación de la ciencia del pasado mediante la modernidad de la ciencia» (Bachelard, 1951a, p. 131), que conlleva la consecuencia de que la historia se esté reescribiendo en forma continua. La historiografía recurrente aspira a lo que Bachelard (1951 a) denomina «historia 
sancionada" (histoire sanctionnée), la cual se opone a la historia tradicionai y «obsoleta» (histoire perimée), que se dedicá simplemente a describir los acontecimientos del pasado. En palabras de Fichant (Fichant y Pécheux, 1971 , p. 96), la historia obsoleta es «la historia de los pensamientos que ya no pueden pensarse en la racionalidad del presente», mientras que la historia sancionada es «la historia de los pensamientos que siguen siendo actuales o que podrian hacerse actuales si se los evalúa según la ciencia de la actualidad». De este modo, Bachelard se distancia tanto del diacronismo más estricto como de la historiografía tradicional, teleológica y continuista.

Si bien el debate aún no ha concluido, la historiografía actual tiende a adoptar una posición intermedia entre anacronismo y diacronismo estrictos, posición que permita la producción de relatos históricos muy variados: el requisito de adoptar a priori un enfoque único y excluyente para todos los casos implica, en sí mismo, un cierto dogmatismo. Como afirma Helge Kragh (1989, p. 147), el historiador de la ciencia, al igual que Jano, deberá poseer los dos rostros que le permitan reconocer las ventajas y admitir las limitaciones de ambos enfoques.

\section{LA HISTORIA COMO INTERPRETACIÓN}

La polémica whig versus antiwhig nos deja una importante enseñanza: no existe una única manera correcta de escribir la historia. Todo relato histórico implica necesariamente interpretación: Ia historia no es meramente narrada, sino construida.

El cuestionamiento de la objetividad absoluta en la producción del relato histórico tiene también antecedentes lejanos. Ya durante la Ilustración, los historiadores compartían la opinión de que todas las representaciones históricas dependen de la elección del autor; tal elección resulta ineludible en la medida en que el historiador se mueve dentro de sus propios límites sociales, religiosos, políticos o ideológicos. En esta línea de pensamiento, el historiador alemán Thomas Abbt afirmaba, ya en 1766 , la posibilidad de que diferentes relatos de los mismos sucesos pudieran resultar igualmente verdaderos (Koselleck, 1993, p. 308).

EI perspectivismo histórico se acentuó aún más cuando adquirió también una dimensión temporal además de la dimensión espacial. Las interpretaciones pueden variar no sólo entre autores contemporáneos, sino desde diferentes "presentes" a lo largo de la historia. De este modo, no sólo se hizo posible, sino incluso necesario, que los mismos acontecimientos fueran narrados y juzgados de manera diferente con el transcurso del tiempo. El propio Goethe fundamentó este requisito de reescribir la historia una y otra vez, no por el hallazgo de nuevas fuentes, Jo cual según la estrategia de la investigación habría resultado evidente; sino por el contrario, porque exigia la continua relectura de la historia universal sobre la base de que los observadores de distintas épocas adoptan puntos de vista desde los cuales es posible apreciar y juzgar el pasado de una forma nueva (Koselleck, 1993 , p. 313). Esto indica que, ya a fines del sigIo XVIII, no se consideraba la relatividad de los juicios históricos como una anomalía de la teoría del conocimiento histórico, sino como testimonio de una verdad superior: el pasado se revela en su verdad respecto del presente desde el cual se observa.

En sus pormenorizadas investigaciones historiográficas, Reinhart Koselleck (1993) contrapone el enfoque medieval a la nueva visión del conocimiento histórico surgida en la modernidad. Los historiadores medievales, con su historiografía aditiva, consideraban como su misión el registro pormenorizado en los anales de todo lo nuevo, acontecimiento por acontecimiento. Con el advenimiento de la modernidad, «Ios acontecimientos perdieron el carácter estable por el que se habían fijado por escrito en los anales y continuaban escribiéndose [...]. Un acontecimiento podia, desde entonces, cambiar su identidad si se modificaba su estatus en la historia total que progresaba continuamente» (Koselleck, 1993, p. 312).

La epistemología actual también reconoce la imposibifidad de una objetividad absoluta en la historia. Toda construcción de un relato histórico implica decisiones por parte del historiador respecto de los factores relevantes para los acontecimientos que desea presentar. Tales decisiones se encuentran fuertemente condicionadas por la perspectiva específica del historiador: su propio contexto histórico-social, su ideología, su cosmovisión. En el caso particular de la historia de la ciencia, un elemento decisivo de la interpretación histórica consiste en la posición epistemológica deł historiador: su concepción filosófica acerca de la ciencia y del método científico, sea conciente o inconcientemente, determina fuertemente la resultante lectura de la historia. En efecto, los historiadores whig suelen sostener la tradicional visión acumulativa y progresiva de la ciencia; el progreso del conocimiento puede ser evaluado con relación a una meta única y transhistórica, que consiste en la obtención de teorías verđaderas. Los historiadores antiwhig, por el contrario, suelen seguir el camino inaugurado por Thomas Kuhn (1970) con stu visión paradigmática de la ciencia: no existe la piedra de toque para comparar las producciones científicas de diferentes épocas; la evolución histórica de la ciencia se despliega en una sucesión de paradigmas inconmensurables.

En definitiva, en la actualidad se reconoce que la búsqueda de una absoluta imparcialidad en historia constituye un mito. Por el contrario, se admite que no sólo la literarura y el resto de las artes tienen su problema hermenéutico; también la historia de la ciencia implica el problema de la interpretación y la cuestión del perspectivismo.

\section{EL CASO DE GALILEO}

Tal vez, el caso de la interpretación de la obra galileana sea el que ilustra con mayor claridad la posibilidad de relatos históricos totalmente disimiles acerca de un mis- 
mo acontecimiento del pasado. Las numerosas y contradictorias lecturas del personaje histórico y de su obra ponen de manifiesto el aspecto constructivo e interpretativo de la historia.

Durante el siglo pasado y en sintonía con la epistemología empirista dominante, prevaleció la imagen de un Galileo empirista que aún puede encontrarse en los textos escolares modelando la lectura más difundida del autor. Según esta interpretación, entre Aristóteles y Galileo sólo habría mediado la tiniebla del medioevo cuya vana filosofía especulativa nada podía aportar a la ciencia y frente a la cual se alzaron los héroes del Renacimiento con su espíritu crítico y su método experimental. Pero a fines del siglo XIX se abrió una nueva perspectiva para los estudios galileanos: la edición autorizada de las obras completas de Galileo a cargo de Antonio Favaro (1909), así como la publicación de los trabajos del raismo editor, permitieron que la investigación profundizara sobre los textos originales del Pisano y abrieron el camino de los primeros cuestionamientos a la concepción de la obra galileana entonces vigente. De este modo, este siglo fue testigo del surgimiento de una serie de importantes estudiosos que reformularon la imagen de Galileo, dando origen a una polémica que, en un sentido general, enfrentó dos corrientes interpretativas: la posición que postula una continuidad entre los trabajos de Galileo y la física medieval tardía -representada por Duhem y Wallace-y la perspectiva que defiende la tesis de la ruptura entre el medioevo y la modernidad-defendida por Koyré y Drake-. A esta polémica se superpone otra referida al peso del componente experimental en los trabajos galileanos: así, a la interpretación empirista tradicional reactualizada por Drake se opone la imagen de un Galileo racionalista-platónico originada en los trabajos de Koyré.

La publicación de los cuadernos de Leonardo da Vinci permitio a Pierre Duhem enfrentarse, a principios de este siglo, a la concepción decimonónica acerca de los orígenes de la ciencia moderna en el Renacimiento. A partir del paciente estudio de manuscritos medievales, Duhem (1959) destruyó la visión tradicional de un vacío científico durante el Medioevo: no sólo sugirió una continuidad entre la física medieval y la renacentista, sino también puso en duda seriamente la originalidad de la obra galileana, afirmando que los logros científicos de Galileo habrían sido anticipados en sus aspectos esenciales por los físicos parisinos del siglo XIV. En este continuo sin rupturas que lleva desde la física medieval a la mecánica galileana, para Duhem, dos nombres merecen ser destacados: Juan Buridan y Nicolás de Oresme. Buridan, con su teoría del ímpetu, formulada para solucionar los problemas de la teoría aristotélica del movimiento forzado, anticipó la noción galileana de inercia; Oresme, mediante sus gráficos de la velocidad en función del tiempo logró una aproximación muy directa al concepto de aceleración y formuló el llamado teorema de la velocidad media, que Galileo utilizó con una notacion muy similar a la original.

La tesis continuista de Duhem, a pesar de basarse en una sólida documentación, se enfrentó con numerosas críti- cas, en particular por parte de los historiadores sociales, quienes sostienen que la ciencia galileana, a diferencia de la medieval, es el producto de una sociedad en la cual la burguesía en ascenso aspira a un conocimiento que conduce al poder técnico para dominar la naturaleza.

La idea de un Galileo racionalista y platónico se inició en la década de 1940 con los trabajos de Alexandre Koyré (1980). A la imagen de un Galileo mecanicista, heredero de la ingeniería alejandrina de Arquímedes y renacentista de Leonardo, que concebía el universo como una máquina y a Dios como el supremo ingeniero, Koyré enfrento la interpretación que convierte al Pisano en un defensor de la tradición neopjatónica, a la manera de Giordano Bruno y Kepler. La utilización recurrente de la matemática por parte de Galileo deja de tener el carácter instrumental que le asignaban los historiadores cmpiristas para convertirse en el medio de captar a priori la esencia y estructura de la realidad, cifrada por Dios en caracteres geométricos. De acuerdo con esta interpretación, Galileo nunca realizó en forma efectiva los experimentos que se le atribuyen y que describe minuciosamente en sus trabajos: según Koyré, tales experimentos fueron únicamente imaginarios o pensados, pues las Iimitaciones del instrumental disponible en la época tornaban imposible la fabulosa exactitud de las supuestas mediciones galileanas. Koyré considera que un aspecto en el que se pone claramente de manifiesto el platonismo galileano consiste en la geometrización del espacio; es decir, la sustitución de la concepción de un espacio cósmico cualitativamente diferenciado y concreto, el de la física pregalileana, por el espacio homogéneo y abstracto de la geometría euclideana. Esta nueva concepción del espacio conduce a una geometrización de la naturaleza y la consiguiente geometrización de la ciencia, e implica el abandono de la noción de lugares privilegiados y movimientos «naturales» o «violentos» dirigidos hacia y a partir de tales lugares. Esta ruptura del cosmos antiguo cerrado sobre sí mismo y la apertura de un universo infinito (Koyré, 1982) es la categoría fundamental de análisis de Koyré, quien, de este modo, rechaza la tesis de continuidad formulada por Duhem señalando una ruptura entre la física medieval y la concepción galileana.

La interpretación đe Koyré entró en crisis en la década de 1960 , cuando una serie de historiadores-experimentadores, provistos de relojes de agua, esferas de bronce y planos inclinados, repitieron los cuestionados experimentos galileanos mostrando su factibilidad con el instrumental disponible en la época. En particular, Thomas Settle (1961) reconstruyó el experimento del plano inclinado centrando su atención en la medición del tiempo: utilizando tratados de la época, mostró que era posible medir el tiempo por medio de relojes de agua con la precisión mencionada por Galíleo en su obra.

Pero quien puso en jaque la imagen exclusivamente racionalista de Galileo fue Stillman Drake (1978). Mientras, hasta el momento, todos los investigadores se circunscribían a la edición de Favaro, Drake introdujo la novedad de tomar en consideración también los 160 
folios de notas y borradores inéditos depositados en la Biblioteca Nacional de Florencia. Mediante el nuevo material, Drake pudo reconstrujr experimentos reales de Galileo hasta entonces desconocidos y comprobar que sus resultados se ajustaban notablemente a los que aparecían documentados. Así, por cjemplo, Drake analizó profundamente el folio 116, en el cual Galileo resume los resultados de un experimento realizado con un plano inclinado sobre una mesa. A partir de una altura determinada podía echarse a rodar una esfera maciza de bronce guiada por una canaleta, en cuyo extremo inferior se fijaba un deflector, de manera que la bola iniciara horizontalmente su caída libre. En el folio pueden observase cinco curvas claramente parabólicas y al lado de cada punto de impacto aparecen dos números: uno señalado con la palabra dovería, que indica cl valor calculado, y el otro que consiste en el resultado de la medición; las diferencias entre los cálculos y las medidas experimentales muestran un error inferior al $4 \%$. Sobre la base de esta minuciosa tarea de investigación, Drake reactualiza la imagen de un Galileo experimentador.

En lo que se refiere a la relación entre la obra galileana y los trabajos de los eruditos parisinos, Drake rechaza explícitamente la tesis de la continuidad iniciada por Duhem, sosteniendo que los estudios de Galileo acerca del movimiento están libres de toda influencia de la física medieval y, por tanto, resultan totalmente originales y autónomos respecto de sus precedentes históricos.

William Wallace (1984), por su parte, si bien adscribe la tesis de la continuidad, a diferencia de Duhem no encuentra Ios antecedentes de Galileo en los eruditos parisinos, sino en la filosofía natural escolástica. Sobre la base de indagaciones histórico-fílosóficas acerca de un nuevo maluscrito galileano descubierto por él mismo, Wallace se opone a Ia interpretación platonizante de Koyré y a la interpretación positivista de Drake. En su obra subraya la necesidad de investigar la relación conceptual entre los escritos lógicos galíleanos y los textos metodológicos de Aristóteles (1984), en especial los Segundos analíticos y algunos pasajes de la Física. Esta necesidad se basa en la principal tesis sostenida por Wallace, según la cual los verdaderos precursores de Galileo no son los autores señalados por Duhem, sino los profesores jesuitas del Colegio Romano pertenecientes a la corriente tomista. Incluso el aspecto metodológico comúnmente considerado como el más original en Galilco-su utilización de la matemática para la descripción de la naturaleza- puede encontrar, según Wallace, su fuente en los trabajos del padre Clavius, profesor jesuita de matemática en el Colcgio Romano, con quien Galileo mantenía una estrecha relación.

\section{CONCLUSIONES}

Luego de este recorrido por diversas cuestiones epistemológicas e historiográficas, es hora de volver a las objeciones al programa de introducir la historia en la enseñanza de ciencias mencionadas al comienzo del artículo. A la luz de las consideraciones presentadas, cl argumento de Klein basado en la simplificación de la historia y el argumento de Whitaker que se funda en el problema de la interpretación de los datos históricos, si bien alertan frente a posibles distorsiones desmesuradas en la presentación del devenir del conocimiento científico, parecen anclarse en una conccpción simplista y, en la actuahidad, anticuada de la propia naturaleza de la historia como disciplina. Tales críticas evocan la imagen de una historia concebida como una actividad destinada a narrar objetivamente los hechos pretéritos con cl fin de descubrir el velo del pasado de una vez y para siempre. De acuerdo con esta perspectiva, toda simplificación constituye un «defecto» del relato histórico; a su vez, la existencia de diferentes versiones de un mismo acontecimiento histórico implica el carácter «crrado» de al menos una de ellas.

Por el contrario, Ia historia es hoy concebida como una disciplina en constante desarrollo, que permite una continua relectura de los episodios octirridos en el pasado. La existencia de una multiplicidad de relatos históricos acerca de un mismo acontecimiento, lejos de representar un «defecto» o un «error», es una característica constitutiva de la propia actividad; es decir, forma parte de la naturaleza misma del conocimiento histórico.

Por lo tanto, la necesidad de simplificación y el problema hermenéutico en la historia de la ciencia no constitu* yen «imperfecciones» que impidan introducir la dimensión histórica en la enseñanza de ciencias. Por el contrario, tales aspectos del conocimiento histórico pucden contribuir a desarrollar en los estudiantes el pensamiento crítico así como introducirlos en el arduo problema de lá interpretación tanto de textos como de hechos. En modo alguno resulta ilegítimo que el docente presente su propio relato histórico, su propia interpretación del pasado, siempre que no pretenda convertir su versión en inobjetable y definitiva; es deber del profesor poner de manifiesto el carácter constructivo de la historia así como su propia posición epistemológica desde la cual elabora su relato. Si se adoptan tales recaudos, la historia de la ciencia deja de ser para el alumno un contenido más, una mera narración de acontecimientos, para convertirse en un poderoso estímulo para la reflexión. En definitiva, a pesar de las críticas de Klein y Whitaker, los argumentos en favor de una perpectiva histórica en la enseñanza de ciencias son tan numerosos y variados que justifican Ios intentos de adoptar este nuevo enfoque. 


\section{REFERENCIAS BIBLIOGRÁFICAS}

ARISTÓTELES (1984). The Complete Works of Aristotle. Jonathan Barnes. Princeton: Princeton University Press.

BACHELARD, G. (195la). L'Actualité de l'Histoire des Sciences. París: Découverte.

BACHELARD, G. (195 tb). L'Activité Rationaliste de la Physique Contemporaine. París: Presses Universitaires de France.

BOIDO, G. (1992). La polémica sobre el enfoque Whig en la historia de la ciencia. Análisis Filosófico, Vol. XII, núm. 2.

BUTTERFIELD, $\mathrm{H} .(195 \mathrm{I})$. The Whig Interpretation of History. Nueva York: Charles Scribner's Sons.

COUSIN, V. (1828). Introduction àl'Histoire de la Philosophie. París: Didier.

DOBBS, B.J.T. (1975). The Foundations of Newton's Alchemy or "The Hunting of the Greene Lyon". Cambridge: Cambridge University Press.

DOBBS, B.J.T. (1991). The Janus Faces of Genius. The Role of Alchemy in Newton's' Thought. Cambridge: Cambridge University Press.

DRAKE, S. (1978). Galileo at Work. Chicago: University of Chicago Press.

DUHEM, P. (1959). Le Systeme du Monde. 10 vol. París: Hermann et Fïs.

FAVARO, A. (ed.)(1909), Le Opere de Galileo Galilei. 20 vol. Florencia: Edizione Nazionale.

FICHANT, M. y PECHEUX, M. (1971). Sobre la historia de la ciencia. Buenos Aires: Siglo XXI (1a. ed. 1969).

FIGALA, K. (1977). Newton as Alchemist. History of Science. Vol. 15, pp. 102-137.

[Artículo recibido en abril de 1996 y aceptado en mayo de 1997.]
HEGEL, G.W.F. (1975). Principios de la filosofía del derecho. Buenos Aires: Sudamericana (1a. ed. 1821).

HEGEL, G.W.F. (1985). Fenomenología del Espíritu. México: Fondo de Cultura Económica (1a. ed. 1807).

KLEIN, M.J. (1972). Use and Abuse of Historical Teaching in Physics, en Brush S.G. y King A.L. (eds.). History in the Teaching of Physics. Hanover: University Press of New England.

KOSELLECK, R. (1993). Futuro pasado. Para una semántica de los tiempos históricos. Barcelona: Paidós (1a. ed. 1979).

KOYRE, A. (1980). Estudios galileanos. Madrid: Siglo XXI (1a. ed. 1940).

KOYRE, A. (1982). Del mundo cerrado al universo infinito. México: Siglo XXI (la. ed. 1957).

KRAGH, H. (1989). Introducción a la historia de la ciencia. Barcelona: Crítica (1a.ed. 1987).

KUHN, T. (1970). The Structure of Scientific Revolutions. Chicago: University of Chicago Press (1a. ed. 1962).

SARMJENTO, D.F. (1993). Facundo. Buenos Aires: Espasa Calpe (1a. ed. 1845).

SARTON, G. (1979). Historia de la ciencia. 3 vol. Buenos Aires: Eudeba.

SETTLE, $\Upsilon$. (1961). An Experiment in the History of Science. Science, Vol. 133, pp. 20-28.

WALLACE, W. (1984), Galileo and his sources. Princeton: Princeton University Press.

WHITAKER, M.A.B. (1979). History and Quasi-History in Physics Education, I y II. Physics Education, Vol. 14, pp. $108-112 ; 239-242$. 
